\title{
ALMA Study of the Lensed Galaxy SDP.81
}

\author{
Jazeel H. Azeez ${ }^{1 *}$, Sadeem Abbas Fadhil ${ }^{1}$, Zainab K. Naser Alla ${ }^{1}$ and Zamri Z. Abidin ${ }^{2}$ \\ ${ }^{1}$ Department of Physics, College of Science, Al-Nahrain University, Baghdad-Iraq. \\ ${ }^{2}$ Department of Physics, College of Science, University of Malaya, Kuala Lumpur-Malaysia. \\ Corresponding Author: jazeelhussein@yahoo.com
}

\begin{abstract}
In this paper we present high resolution data from Atacama Large Millimeter /sub-millimeter Array (ALMA) of CO (5-4) and CO (8-7) rotational transitions toward the gravitational lensed galaxy (SDP.81). We calculate the molecular gas mass in the clumpy region which is around $1.5 \times 10^{9} \mathrm{M}_{\odot}$. We overly the $\mathrm{CO}(8-7)$ and $\mathrm{CO}(5-4)$ intensity distributions to calculate the brightness temperature ratio $\mathrm{R}_{85}$ at various region. The $\mathrm{CO}$ line ratio was in the range between 0.39 and 0.75 with an average value about 0.48 . This line ratio suggests that most sub-millimeter galaxies, SMGs, include a significant proportion of extended, moderate-density, cool gas. [DOI:
\end{abstract} 10.22401/ANJS.00.1.09]

Keywords: Galaxies, High redshift, ALMA, SDP.81.

\section{Introduction}

Gravitational lensing is an important astrophysical tool to understand the formation and evolution of distant galaxies. At submillimeter wavelength, the investigation of redshifted molecular lines in gravitationally lensed sources provides a valuable diagnostic tracer of their interstellar medium, structure and dynamics [1-4]. H-ATLAS J090311.6+ 003906 (also known as SDP.81) is a gravitationally lensed sub-millimeter galaxy (SMG) at redshift $\mathrm{z}=3.042$ that was discovered in Herschel Astrophysical Terahertz Large Area Survey [5, 6].

Obtaining the desired high-resolution imaging in the sub-millimeter become available using ALMA. The aim of this manuscript is to use the high-resolution ALMA imaging of the lensed source to study the physical properties of its molecular gas. The structure of this manuscript is composed from the following sections: Section 2 describe the radio instrument. Section 3.1 describe the data and initial results. In section 3.2 we calculate the brightness temperature ratio. Lastly, the summary and conclusions were listed in section 4 .

\section{Radio Instruments}

ALMA is the largest astronomical project to date. It is composed of a large array of $12 \mathrm{~m}$ antennas, with baselines of up to $16 \mathrm{~km}$. The antennas are movable within the surrounding regions. In addition to the $12 \mathrm{~m}$ array, ALMA is also equipped with the Atacama Compact Array (ACA), which is composed of twelve 7 $\mathrm{m}$ antennas and four $12 \mathrm{~m}$ antennas. The ACA array is often fixed and used for observing large-scale structures that are not clearly imaged by the $12 \mathrm{~m}$ array. Extra extended arrays can offer higher spatial resolutions, whereas more compact arrays can offer the best sensitivity for extended objects.

\section{Results and Discussion \\ 3.1 Molecular Gas Mass}

SDP.81 was observed with ALMA science verification SV data band 4 on 2014 October 21 . The band 4 receivers were tuned to cover $\mathrm{CO} \mathrm{J}=5-4$ transition (rest frequency = $576.267 \mathrm{GHz}$; SDP.81's redshift=3.042). The observational characteristics are shown in Table (1). The data were manipulated using the Common Astronomy Software Application (CASA). We have used the publically available SV CO $(\mathrm{J}=5-4)$ data as a tracer for molecular gas mass. The $\mathrm{CO}(\mathrm{J}=5-4)$ integrated intensity and velocity field maps are shown in Fig.(1). 
Table (1)

ALMA Observational Characteristics.

\begin{tabular}{|c|c|}
\hline Characteristic & Value \\
\hline Galaxy & $\begin{array}{c}\text { H-ATLAS J090311.6+003906 } \\
\text { (SDP.81) } \\
\end{array}$ \\
\hline Observing date & 2014 October 21 \\
\hline $\begin{array}{l}\text { Field center: } \\
\text { R.A. }\left(\mathbf{J}_{2000}\right) \\
\text { Dec. }\left(\mathbf{J}_{2000}\right) \\
\end{array}$ & $\begin{array}{l}09^{\mathrm{h}} 03^{\mathrm{m}} 11.610^{\mathrm{s}} \\
+00^{\circ} 39^{\prime} 06.700^{\prime \prime}\end{array}$ \\
\hline Rest frequency & $576.267 \mathrm{GHz}$ \\
\hline $\begin{array}{l}\text { Velocity } \\
\text { resolution }\end{array}$ & $21 \mathrm{~km} \mathrm{~s}^{-1}$ \\
\hline Restoring beam & $\begin{array}{l}\text { (major, minor, P.A.) } \\
0.15^{\prime \prime}, 0.12^{\prime \prime}, 57.26\end{array}$ \\
\hline
\end{tabular}

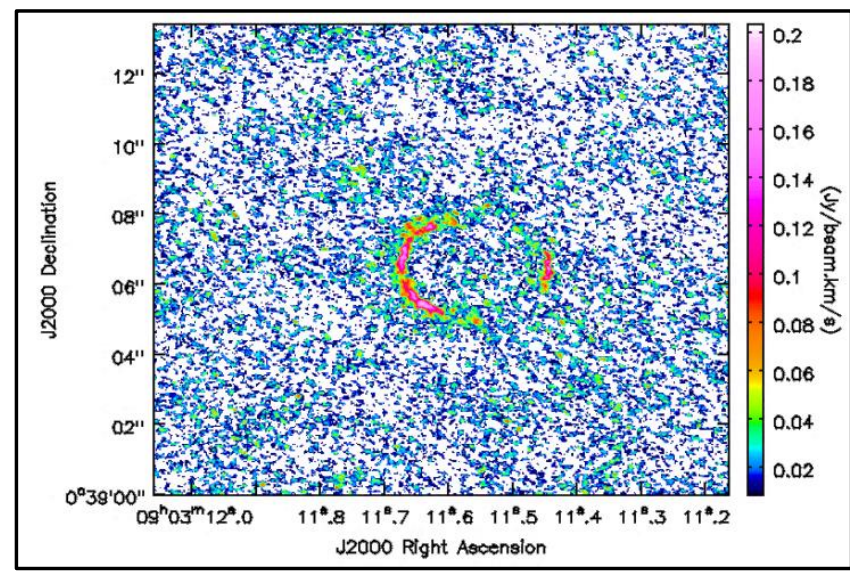

Fig.(1) ALMA CO $(J=5-4)$ integrated intensity image of SDP.81.

We calculate the molecular gas mass using the following formula [7]:

$\left(\frac{M_{\mathrm{H}_{2}}}{M_{\odot}}\right)=\alpha_{C O} L_{C O}^{\prime}$

where $\alpha_{C O}$ is CO-to- $\mathrm{H}_{2}$ gas mass conversion factor which is equal to $0.8 M_{\odot}(\mathrm{K} \mathrm{km}$ $\left.\mathrm{s}^{-1} \mathrm{pc}^{2}\right)^{-1}$ [8], $L_{C O}^{\prime}$ is CO luminosity which was calculated from the following equation:

$L_{C O}^{\prime}=3.25 \times 10^{7} S_{C O} \Delta v v_{o b s}^{-2} D_{L}^{2}(1+z)^{-3}$

where $S_{C O} \Delta v$ is the intensity of ${ }^{12} \mathrm{CO}(\mathrm{J}=5-4)$ in Jy $\mathrm{km} \mathrm{s}^{-1}, \mathrm{D}_{\mathrm{L}}$ is the luminosity distance in $\mathrm{Mpc}$ and $v_{o b s}^{-2}$ is the observed frequency in GHz. We adopted CO line ratio $\mathrm{R}_{51}=0.3$, [9]. The total molecular gas mass $\mathrm{M}\left(\mathrm{H}_{2}\right)$ within the area of clumpy molecular gas $\Delta x \times \Delta y=4^{\prime \prime} \times 4^{\prime \prime}$ is equal to $1.5 \times 10^{9} \mathrm{M}_{\odot}$.

\subsection{CO Line Ratio}

In Fig.(2) we overlay ALMA integrated intensity maps of the $\mathrm{CO}(\mathrm{J}=8-7)$ line emission (raster image) and $\mathrm{CO}(\mathrm{J}=5-4)$ line emission (contour image) for the galaxy SDP.81. We can see from this figure the overall distribution of both $\mathrm{CO}$ (8-7) and $\mathrm{CO}$ (5-4) are very similar. We divided the clumpy molecular gas into 8 regions $(1 \rightarrow 8)$ in the same way as $[10$, $11,12]$. The size of each region is $0.5^{\prime \prime} \times 0.5^{\prime \prime}$. The $\mathrm{CO}(8-7) / \mathrm{CO}(5-4)$ brightness temperature ratios, $\mathrm{R}_{85}$, are calculated for each region and listed in Table (2). There is no clear ratio difference between these 8 regions which have a range of brightness temperature ratio between $0.39-0.75$. The average value in these regions about 0.48 . This value was consistent with previous work. The values of the line ratios propose that most sub-millimeter galaxies, SMG, include a significant proportion of extended, moderate-density, cool gas, [13].

\section{Table (2)}

The $\mathrm{CO}(8-7) / C O(5-4)$ Line Ratios in Different Areas.

\begin{tabular}{|c|c|c|c|}
\hline Region & R.A. $\left(\mathbf{J}_{2000}\right)$ & Dec. $\left(\mathbf{J}_{2000}\right)$ & $\mathbf{R}_{\mathbf{8 5}}$ \\
\hline 1 & 09:03:11.640 & $+00: 39: 07.648$ & 0.52 \\
\hline 2 & 09:03:11.668 & $+00: 39: 07.070$ & 0.39 \\
\hline 3 & 09:03:11.673 & $+00: 39: 06.507$ & 0.43 \\
\hline 4 & 09:03:11.665 & $+00: 39: 05.957$ & 0.36 \\
\hline 5 & 09:03:11.649 & $+00: 39: 05.394$ & 0.45 \\
\hline 6 & 09:03:11.612 & $+00: 39: 05.302$ & 0.49 \\
\hline 7 & 09:03:11.446 & $+00: 39: 06.767$ & 0.75 \\
\hline 8 & 09:03:11.442 & $+00: 39: 06.225$ & 0.45 \\
\hline
\end{tabular}

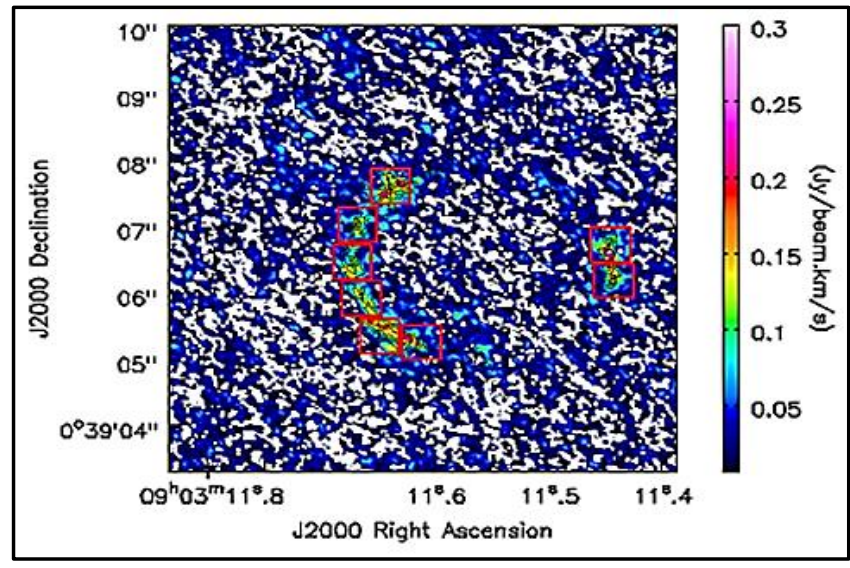

Fig. (2) ALMA integrated intensity map of the $\mathrm{CO}(\mathrm{J}=5-4)$ line emission (contour image) overlaid on $\mathrm{CO}(\mathrm{J}=8-7)$ line emission (raster image) for the galaxy SDP.81. We also plot 8 
boxes $(1 \rightarrow 8)$ that are used to calculate the line ratios.

\section{Summary and Conclusions}

We used a very high resolution data of ALMA observation of the ${ }^{12} \mathrm{CO}(\mathrm{J}=8-7)$ and ${ }^{12} \mathrm{CO}(\mathrm{J}=5-4)$ line emissions as a molecular gas indicator in the clumpy region of the gravitational lensed galaxy H-ATLAS J090311.6+003906 (SDP.81) at redshift $\mathrm{z}=$ 3.042. Both the ${ }^{12} \mathrm{CO}(\mathrm{J}=8-7)$ and ${ }^{12} \mathrm{CO}(\mathrm{J}=5-4)$ line show similar distribution in the integrated intensity maps. We find the molecular gas mass from ${ }^{12} \mathrm{CO}(\mathrm{J}=5-4)$ equal to $1.5 \times 10^{9} \mathrm{M}_{\odot}$. We also find the brightness temperature ratio $\mathrm{R}_{85}$ at different regions. The $\mathrm{CO}$ line ratio varies between 0.39 and 0.75 with an average value of 0.48 . This value of line ratio suggests that most of the SMGs have a considerable amount of extended, moderate-density, cool gas.

\section{Acknowledgments}

This paper makes use of the following ALMA data: ADS/JAO.ALMA \#2011.0. 000016.SV. ALMA is a partnership of ESO (representing its member states), NSF (USA) and NINS (Japan), together with NRC (Canada) and NSC and ASIAA (Taiwan), and KASI (Republic of Korea), in cooperation with the Republic of Chile. The Joint ALMA Observatory is operated by ESO, AUI/NRAO and NAOJ.

\section{References}

[1] Swinbank, A. M., Smail, I., Longmore, S., et al., "Intense star formation within resolved compact regions in a galaxy at $\mathrm{z}=$ 2.3", Nature, 464, 733-736, 2010.

[2] Swinbank, A. M., Papadopulos, P. P., Cox, P., et al., "The Interstellar Medium in Distant Star-forming Galaxies: Turbulent Pressure, Fragmentation, and Cloud Scaling Relations in a Dense Gas Disk at z $=2.3$ ”, ApJ, 742, 11-23, 2011.

[3] Riechers, D. A., Cooray, A., Omont, A., et al., "Dynamical Structure of the Molecular Interstellar Medium in an Extremely Bright, Multiply Lensed $\mathrm{z} \sim=3$ Submillimeter Galaxy Discovered with Herschel", ApJ, 733, 12-17, 2011
[4] Fu, H., Cooray, A., Feruglio, C., et al., "The rapid assembly of an elliptical galaxy of 400 billion solar masses at a redshift of 2.3", Nature, 498, 338-341, 2013.

[5] Eales, S., Dunne, L., Clements, D., et al., "The Herschel ATLAS", PASP, 122, 499, 2010.

[6] Negrello, M., Hopwood, R., de Zotti, G., et al., "The Detection of a Population of Submillimeter-Bright, Strongly Lensed Galaxies", Sci, 330, 800, 2010.

[7] Solomon, P. M., \& Vanden Bout, P. A., "Molecular Gas at High Redshift", ARA\&A, 43, 677, 2005.

[8] Downes, D., \& Solomon, P. M., "Rotating Nuclear Rings and Extreme Starbursts in Ultraluminous Galaxies", ApJ, 507, 615, 1998.

[9] ALMA Partnership, Vlahakies, C., Hunter, T. R. et al., "The 2014 ALMA Long Baseline Campaign: Observations of the Strongly Lensed Submillimeter Galaxy HATLAS J090311.6+003906 at z = 3.042”, ApJ, 808, L4, 2015.

[10] Tsai, M., Hwang, C. Y., Matsushita, S., Baker, A. J., \& Espada, E., "Interferometric $\mathrm{CO}(3-2)$ Observations toward the Central Region of NGC 1068", ApJ, 746, 129, 2012.

[11] Azeez, J. H., Hwang, C. Y., Abidin, Z. Z., et al., "Kennicutt-Schmidt Law in the Central Region of NGC 4321 as Seen by ALMA”, Sci. Rep. 6, 26896, 2016.

[12] Jazeel H. Azeez, Zamri Z. Abidin, C.-Y. Hwang, and Zainol A. Ibrahim, "Star Formation Law at Sub-kpc Scale in the Elliptical Galaxy Centaurus A as Seen by ALMA", Advances in Astronomy, 2017, Article ID 8416945, 2017.

[13] Ivison, R. J., Papadopoulos, P. P., Smail, I., et al., "Tracing the molecular gas in distant submillimetre galaxies via $\mathrm{CO}(1-0)$ imaging with the Expanded Very Large Array", MNRAS, 412, 1913, 2011. 
Jazeel H. Azeez 